PROCEEDINGS OF THE

AMERICAN MATHEMATICAL SOCIETY

Volume 127, Number 2, February 1999, Pages 543-551

S 0002-9939(99)04887-X

\title{
ON SMOOTHNESS OF CARRYING SIMPLICES
}

\author{
JANUSZ MIERCZYŃSKI
}

(Communicated by Hal L. Smith)

\begin{abstract}
We consider dissipative strongly competitive systems $\dot{x}_{i}=x_{i} f_{i}(x)$ of ordinary differential equations. It is known that for a wide class of such systems there exists an invariant attracting hypersurface $\Sigma$, called the carrying simplex. In this note we give an amenable condition for $\Sigma$ to be a $C^{1}$ submanifold-with-corners. We also provide conditions, based on a recent work of M. Benaïm (On invariant hypersurfaces of strongly monotone maps, J. Differential Equations 136 (1997), 302-319), guaranteeing that $\Sigma$ is of class $C^{k+1}$.
\end{abstract}

\section{INTRODUCTION}

We consider systems of ordinary differential equations (ODE's) of class (at least) $C^{1}$

$$
\dot{x}_{i}=x_{i} f_{i}(x)
$$

on the nonnegative orthant $C:=\left\{x \in \mathbb{R}^{n}: x_{i} \geq 0\right.$ for $\left.1 \leq i \leq n\right\}, n \geq 3$.

We write $F_{i}(x)=x_{i} f_{i}(x), F=\left(F_{1}, \ldots, F_{n}\right)$. The symbol $D F=\left[\partial F_{i} / \partial x_{j}\right]_{i, j=1}^{n}$ stands for the derivative matrix of the vector field $F$. The local flow generated by (E) on $C$ will be denoted by $\phi=\left\{\phi_{t}\right\}$. A subset $B \subset C$ is invariant [resp. forward invariant] if $\phi_{t} x \in B$ for all $(t, x) \in \mathbb{R} \times B$ [resp. for all $\left.(t, x) \in[0, \infty) \times B\right]$ for which $\phi_{t} x$ is defined. For $x \in C, B \subset C$ the symbols $\omega(x), \alpha(x), \omega(B), \alpha(B)$ have their usual meanings (see e.g. Hale [3]). A point $x \in C$ is a rest point if $\phi_{t} x=x$ for each $t \in \mathbb{R}$ (alternatively, if $F(x)=0$ ). An invariant subset $B$ of a compact invariant set $S$ is called an attractor (resp. a repeller) relative to $S$ if there is a relative neighborhood $U$ of $B$ in $S$ such that $\omega(U)=B$ (resp. $\alpha(U)=B$ ). For an attractor $B$ relative to $S$, by the repeller complementary to $B$ we understand the set $\{x \in S: \omega(x) \cap B=\emptyset\}$. The attractor complementary to a repeller $R$ is defined in an analogous way.

System (E) is dissipative if there is a compact set $B \subset C$ such that for each bounded $D \subset C$ its $\omega$-limit set $\omega(D)$ is a nonempty subset of $B$. By standard results on global attractors (see [3]), for a dissipative system (E) there exists a compact invariant set $\Gamma \subset C$ (the global attractor for $(\mathrm{E})$ ) such that $\omega(D) \subset \Gamma$ for each bounded $D \subset C$. Evidently, $0 \in \Gamma$.

Received by the editors June 2, 1997.

1991 Mathematics Subject Classification. Primary 34C30, 34C35; Secondary 58F12, 92D40.

The author's research was supported by KBN grant 2 P03A 07608 (1995-97).

(c)1999 American Mathematical Society 
For $I \subset\{1, \ldots, n\}$ denote

$$
\begin{aligned}
C_{I} & :=\left\{x \in C: x_{i}=0 \text { for } i \in I\right\}, \\
C_{I}^{\circ} & :=\left\{x \in C_{I}: x_{j}>0 \text { for } j \notin I\right\}, \\
\partial C_{I} & :=C_{I} \backslash C_{I}^{\circ} .
\end{aligned}
$$

From the form of (E) it follows readily that any $C_{I}$, as well as $\partial C_{I}$ and $C_{I}^{\circ}$, is invariant. We denote by $(\mathrm{E})_{I}$ the restriction of system $(\mathrm{E})$ to $C_{I}$. Instead of $C_{\emptyset}^{\circ}$, $\partial C_{\emptyset}$, we write $C^{\circ}, \partial C . I^{\prime}$ means $\{1, \ldots, n\} \backslash I$.

If system (E) is dissipative, so are all of its subsystems $(\mathrm{E})_{I}$. For each $I \subset$ $\{1, \ldots, n\}$, the global attractor $\Gamma_{I}$ for $(\mathrm{E})_{I}$ equals $\Gamma \cap C_{I}$.

System (E) is called strongly competitive if $\left(\partial f_{i} / \partial x_{j}\right)(x)<0$ for each $1 \leq i, j \leq$ $n, i \neq j, x \in C$. A strongly competitive system is called totally competitive if $\left(\partial f_{i} / \partial x_{i}\right)(x)<0$ for $1 \leq i \leq n, x \in C$. Such systems describe a community of $n$ interacting species where the growth of each species inhibits the growth of any other.

Throughout the rest of the paper the standing assumption will be:

(E) is a $C^{1}$ dissipative strongly competitive system of ODE's satisfying the following:

1. $\{0\}$ is a repeller relative to $\Gamma$.

2. At each rest point $x \in C \backslash\{0\}$ one has $\left(\partial f_{i} / \partial x_{i}\right)(x)<0$ for $1 \leq i \leq n$.

The following important result was established by M. W. Hirsch ([4]).

Proposition 1.1. The attractor $\Sigma \subset \Gamma$ complementary to the repeller $\{0\}$ is homeomorphic via radial projection to the standard $(n-1)$-simplex $\Delta:=\{x \in C$ : $\left.x_{1}+\cdots+x_{n}=1\right\}$. Moreover, the global attractor $\Gamma$ equals the convex hull of $\Sigma \cup\{0\}$.

Following M. L. Zeeman [15], the invariant compact set $\Sigma$ is referred to as the carrying simplex for (E). In the ecological interpretation, the carrying simplex can be thought of as expressing the balance between the growth of small populations ( $\{0\}$ is a repeller) and the competition of large populations (dissipativity).

M. W. Hirsch in [4] asked about sufficient conditions for the carrying simplex $\Sigma$ to be of class $C^{1}$. The time reverse flow $\left\{\phi_{-t}\right\}_{t \geq 0}$ restricted to the invariant set $C^{\circ}$ is strongly monotone and its derivative flow is strongly positive (for these terms see H. L. Smith's monograph [12]). Therefore, when (E) possesses a repeller $R \subset \Sigma \cap C^{\circ}$ relative to $\Sigma$ we can utilize a powerful recent result of I. Tereščák [13] on nonmonotone manifolds to conclude that the repulsion basin $B(R):=\left\{x \in \Sigma^{\circ}\right.$ : $\alpha(x) \subset R\}$ is a $C^{1}$ hypersurface. However, even in that case Tereščák's theorem does not apply to the whole of $\Sigma$, for the time reverse flow fails to be strongly monotone on the boundary $\partial C$. Moreover, if we assume that (E) is permanent (a natural assumption from the applied viewpoint) then there is an attractor $A$ having the whole $C^{\circ}$ as its attraction basin, hence its repulsion basin (relative to $\Sigma$ ) equals $A$. In his paper [10] the present author gave a fairly weak condition implying the $C^{1}$ smoothness of $\Sigma$. It was done, however, at the expense of making use (for $n \geq 5$ ) of Pesin's theory of invariant measurable families of embedded manifolds, which compels one to assume that $f$ has Hölder continuous derivatives.

In this note we show that a well-known, robust, and readily testable condition (see (A)) is enough to conclude that $\Sigma$ is $C^{1}$. Because our proofs exploit Oseledets' theory of Lyapunov exponents, it suffices to assume $f$ is $C^{1}$ to get $C^{1}$ smoothness 
of $\Sigma$. Next, conditions are given, based on recent results of M. Benaïm [1], for the carrying simplex to possess higher order smoothness.

I would like to thank Michel Benaïm for sending me a preprint of [1].

\section{Statement of MAin Results}

For $I \subset\{1, \ldots, n\}$ put

$$
\Sigma_{I}:=C_{I} \cap \Sigma, \quad \Sigma_{I}^{\circ}:=C_{I}^{\circ} \cap \Sigma, \quad \partial \Sigma_{I}:=\partial C_{I} \cap \Sigma .
$$

We will call $\Sigma_{I}$ a $k$-dimensional face of $\Sigma$, where $k=n-1$ - card $I$. Evidently all $\Sigma_{I}$, as well as $\Sigma_{I}^{\circ}$ and $\partial \Sigma_{I}$, are invariant. For $I \subset\{1, \ldots, n\}$, the face $\Sigma_{I}$ is the carrying simplex for subsystem $(\mathrm{E})_{I}$. The 0 -dimensional face $\Sigma_{i^{\prime}}$ consists of a single rest point $x^{(i)}=\left(0, \ldots, 0, x_{i}^{(i)}, 0, \ldots, 0\right)$ with $x_{i}^{(i)}>0$ (called the $i$-th axial rest point).

Let $V=\left\{v=\left(v_{1}, \ldots, v_{n}\right): v_{i} \in \mathbb{R}\right\}$ stand for the vector space of all free $n$-dimensional vectors (in particular, we write the tangent bundle of the orthant $C$ as $T C=C \times V)$. Depending on the context, $\|\cdot\|$ may mean the Euclidean norm of a vector, or the operator norm of a matrix, associated with the Euclidean norm. For $I \subset\{1, \ldots, n\}$, we denote

$$
V_{I}:=\left\{v \in V: v_{i}=0 \text { for } i \in I\right\} .
$$

For any two points $x, y \in C_{I}$, we write $x \leq_{I} y$ if $x_{i} \leq y_{i}$ for all $i \in I^{\prime}$, and $x<_{I} y$ if $x \leq_{I} y$ and $x \neq y$. Moreover, $x \ll_{I} y$ if $x_{i}<y_{i}$ for all $i \in I^{\prime}$. For $I=\emptyset$ we write simply $\leq,<, \ll$. The reversed symbols are used in the obvious way. As each $\left(C_{I}, \leq_{I}\right)$ is a lattice, we can define, for $I \subset\{1, \ldots, n\}$ with card $I \leq n-1$

$$
x^{[I]}:=\bigvee_{i \in I^{\prime}} x^{(i)},
$$

where it is easy to see that $x^{[J]}<_{I} x^{[I]}$ for $I \subsetneq J$.

The following result probably belongs to the folklore in the theory of competitive systems, but I have not been able to locate its proof.

Lemma 2.1. For each $I \subset\{1, \ldots, n\}$ with $1 \leq \operatorname{card} I \leq n-2$ we have $y<_{I} x^{[I]}$ for all $y \in \Sigma_{I}$.

Proof. Suppose to the contrary that there is $y \in \Sigma_{I}$ not in the $<_{I}$ relation to $x^{[I]}$. Assume first that $y=x^{[I]}$, that is, $x^{[I]} \in \Sigma_{I}$. For $i \in I^{\prime}, j \in I^{\prime}, i \neq j$, we have $x_{j}^{[I]}>x_{j}^{(i)}=0$. As $f_{i}\left(x^{(i)}\right)=0$, it follows by strong competitiveness that $f_{i}\left(x^{[I]}\right)<0$ for $i \in I^{\prime}$. Therefore we have $F_{i}\left(x^{[I]}\right)=x_{i}^{[I]} f_{i}\left(x^{[I]}\right)<0$ for all $i \in I^{\prime}$. Consequently, $\phi_{t} x^{[I]} \ll_{I} x^{[I]}$ for $t>0$ sufficiently small. But $\Sigma_{I}$ is invariant, so $\phi_{t} x^{[I]} \in \Sigma_{I}$ for all $t>0$. We have thus obtained two points in $\Sigma_{I}$ related by $\ll_{I}$, which contradicts Lemma 2.5 in Hirsch [4]. Assume that $y \in \Sigma_{I}$ is not in the $\leq_{I}$ relation to $x^{[I]}$. Take an index $k$ for which $y_{k}>x_{k}^{[I]}$. Let $J \subset\{1, \ldots, n\}$ stand for the set of those indices $j$ for which $y_{j}=0$. Evidently $k \in J^{\prime}$ and $I \subset J$. We have $y \in \Sigma_{I} \cap C_{J}^{\circ}=\Sigma \cap C_{I} \cap C_{J}^{\circ}=\Sigma \cap C_{J}^{\circ}=\Sigma_{J}^{\circ}$. As a consequence, $y_{j}>x_{j}^{(k)}=0$ for $j \in J^{\prime}, j \neq k$, and $y_{k}>x_{k}^{[I]}=x_{k}^{(k)}($ since $k \notin I)$. But this means that $y \gg_{J} x^{(k)}$. As both these points are in $\Sigma_{J}$, this again is in contradiction to Lemma 2.5 in [4].

We say (E) satisfies hypothesis (A) if

For each $1 \leq i \leq n$ one has $f_{i}\left(x^{[i]}\right) \geq 0$. 
In light of the strong competitiveness, (A) can be equivalently formulated as: For each $I \subset\{1, \ldots, n\}$ with $1 \leq \operatorname{card} I \leq n-1$ one has $f_{i}\left(x^{[I]}\right) \geq 0$ for $i \in I$.

Hypothesis (A) is well known in the literature on mathematical ecology. Consider the Lotka-Volterra competitive system

$$
\dot{x}_{i}=x_{i}\left(b_{i}-\sum_{j=1}^{n} a_{i j} x_{j}\right),
$$

with $b_{i}>0, a_{i j}>0$. For $(2.1)$ the $i$-th axial rest point is given by $x_{i}^{(i)}=b_{i} / a_{i i}$. It is easy to see that $(\mathrm{A})$ is now equivalent to

$$
b_{i} \geq \sum_{\substack{j=1 \\ j \neq i}}^{n} a_{i j} \frac{b_{j}}{a_{j j}} \quad \text { for each } 1 \leq i \leq n .
$$

We are now in a position to state our main result.

Theorem A. Assume that (E) satisfies (A). Then the carrying simplex $\Sigma$ is a $C^{1}$ submanifold-with-corners neatly embedded in $C$.

For submanifolds-with-corners their neat embeddings, see [10].

We now state some consequences of hypothesis (A). System $(\mathrm{E})_{I}$ is called permanent if there is $\epsilon>0$ such that $\liminf _{t \rightarrow \infty} \rho\left(\phi_{t} x, \partial C_{I}\right) \geq \epsilon$ for each $x \in C_{I}^{\circ}$, where $\rho$ stands for the Euclidean distance between a point and a set.

Proposition 2.2. If (A) is satisfied, then each of the subsystems $(\mathrm{E})_{I}$ is permanent.

Proof. In order not to encumber our presentation with too many subscripts, we prove the assertion for $I=\emptyset$, that is, for system (E) only. For each $i, 1 \leq i \leq n$, we have as a result of strong competitiveness and Lemma 2.1 that $f_{i}(x)>0$ for all $x \in \Sigma_{i^{\prime}}$. Now take a neighborhood $U_{i}$ of $\Sigma_{i^{\prime}}$ in $C$ of the form

$$
U_{i}=\left\{\left(x_{1}, \ldots, x_{n}\right): 0 \leq x_{i}<\epsilon_{i},\left(x_{1}, \ldots, x_{i-1}, x_{i+1}, \ldots, x_{n}\right) \in \tilde{U}_{i}\right\},
$$

where $\epsilon_{i}>0$ and a relative neighborhood $\tilde{U}_{i}$ of $\Sigma_{i^{\prime}}$ in $C_{i^{\prime}}$ are so small that $f_{i}(x)>0$ for all $x \in U_{i}$. As $\Gamma$ is the global attractor for (E) and $\Sigma$ is the attractor relative to $\Gamma$ complementary to $\{0\}$, there exists a forward invariant neighborhood $U$ of $\Sigma$ in $C$ with the property that $\phi_{t} x \in U$ for $x \in C \backslash\{0\}$ and sufficiently large $t$. Also, $U$ can be taken so small that all the sets $\left\{x \in U: x_{i}<\epsilon_{i}\right\}$ are contained in $U_{i}$. Now observe that for $t$ so large that $\phi_{t} x$ belongs to $U$ one has

$$
\frac{d\left(\phi_{t} x\right)_{i}}{d t}=F_{i}\left(\phi_{t} x\right)=x_{i} f_{i}\left(\phi_{t} x\right)>0
$$

as long as $\left(\phi_{t} x\right)_{i}<\epsilon_{i}$. From this it readily follows that $\liminf _{t \rightarrow \infty} \rho\left(\phi_{t} x, \Sigma_{i^{\prime}}\right) \geq \epsilon_{i}$ for any $x \in C^{\circ}$.

In view of results on attractors contained in Hale [3] we have the following.

Lemma 2.3. Under the assumptions of Proposition 2.2, for each $I \subset\{1, \ldots, n\}$ the invariant compact set $\partial \Sigma_{I}$ is a repeller relative to $\Sigma_{I}$.

For $I \subset\{1, \ldots, n\}$ denote by $A_{I}$ the attractor (relative to $\Sigma_{I}$ ) complementary to $\partial \Sigma_{I}$. As $A_{I}$ can be viewed as the global attractor for the semiflow $\left\{\phi_{t}\right\}_{t \geq 0}$ restricted to the connected metric space $\Sigma_{I}^{\circ}$, a result of Gobbino and Sardella (Thm. 3.1 in [2]) yields that $A_{I}$ is connected. 
The ecological interpretation of the property described in Proposition 2.2 is as follows. In each subcommunity none of the species goes extinct, and invasion of a proper subcommunity by others causes the populations of the previously present species to shrink due to the larger amount of competition.

Before formulating sufficient conditions for $\Sigma$ to be of class $C^{k+1}$ we need to introduce some notation (we follow Benaïm's paper [1]). For $x \in A_{I}, I \subset\{1, \ldots, n\}$ with card $I \leq n-2$, we denote by $\lambda(x)$ the largest eigenvalue of the symmetrization of the matrix $\left(-D F^{I}(x)\right)$, where $D F^{I}:=\left[\partial F_{i} / \partial x_{j}\right]_{(i, j) \in I^{\prime} \times I^{\prime}}$. Further, $d(x)$ stands for the square root of

$$
\min _{\substack{i \neq j \\ i, j \notin I}} \frac{\partial F_{i}}{\partial x_{j}}(x) \frac{\partial F_{j}}{\partial x_{i}}(x) .
$$

Put $\lambda_{I}:=\sup \left\{\lambda(x): x \in A_{I}\right\}$ and $d_{I}:=\inf \left\{d(x): x \in A_{I}\right\}$.

We say that (E) satisfying (A) fulfills (C) if for each $I$ with $0 \leq \operatorname{card} I \leq n-2$ any one of the conditions $(\mathrm{C} 1)$ or $(\mathrm{C} 2)$ holds:

(C1) $k \sup \left\{\left\|D F^{I}(x)\right\|: x \in A_{I}\right\}<2(k+1) d_{I}$.

(C2) $k \lambda_{I}<2(k+1) d_{I}$.

Theorem B. Assume that a $C^{k+1}$ system (E) satisfies (A) and (C). Then the carrying simplex $\Sigma$ is a $C^{k+1}$ submanifold-with-corners.

\section{Proof of Theorem A}

Let $\mathbb{S}$ be the $(n-1)$-dimensional sphere $\{v \in V:\|v\|=1\}$. For a vector subspace $W$ of $V$ and $0 \leq k \leq \operatorname{dim} W$, the symbol $\mathbb{G}_{k} W$ denotes the compact metrizable space of all $k$-dimensional vector subspaces of $W$, endowed with the standard topology: for any two $Z_{1}, Z_{2} \in \mathbb{G}_{k} W$, their distance is defined as the Hausdorff distance between $Z_{1} \cap \mathbb{S}$ and $Z_{2} \cap \mathbb{S}$.

The linearization of $(\mathrm{E})$ generates on $T C$ a linear skew-product (local) flow $\left(\phi_{t} x, D \phi_{t}(x) v\right)$, where $D \phi_{t_{0}}(x) v_{0}$ is the value at time $t_{0}$ of the solution of the variational equation $\dot{\xi}=D F\left(\phi_{t} x\right) \xi$ with initial condition $\xi(0)=v_{0}$.

For a linear subset $\mathcal{C}$ of the product bundle $B \times W$, where $B \subset \Sigma$ and $W$ is a vector subspace of $V$, we will denote by $\mathcal{C}_{x}$ the set of all those $v \in W$ such that $(x, v) \in \mathcal{C}$ (in other words, $\{x\} \times \mathcal{C}_{x}$ is the fiber of $\mathcal{C}$ over $\left.x\right)$. A linear subset $\mathcal{C}$ of $B \times W$ is called invariant if for each $(x, v) \in \mathcal{C}$ and each $t \in \mathbb{R}$ one has $\left(\phi_{t} x, D \phi_{t}(x) v\right) \in \mathcal{C}$.

Denote the set of all ergodic measures supported on a compact invariant $B \subset \Sigma$ by $\mathbf{M}_{\text {erg }}(B)$. The multiplicative ergodic theorem of Oseledets (see e.g. Mañé [8]) assures us that if $B \times W$ is an invariant bundle, then for each $m \in \mathbf{M}_{\operatorname{erg}}(B)$ there exist an invariant $m$-measurable set $B_{\text {reg }} \subset B$ (the set of regular points), a collection $\mathcal{C}_{1}, \ldots, \mathcal{C}_{l}$ of invariant linear subsets given by $m$-measurable maps $B_{\text {reg }} \ni$ $x \mapsto\left(\mathcal{C}_{k}\right)_{x} \in \mathbb{G}_{d_{k}} W$ (the Oseledets decomposition) and a collection $\Lambda_{1}<\cdots<\Lambda_{l}$ of reals (Lyapunov exponents) such that

1. $W=\bigoplus_{k=1}^{l}\left(\mathcal{C}_{k}\right)_{x}$ for $x \in B_{\mathrm{reg}}$,

2.

$$
\lim _{t \rightarrow \pm \infty} \frac{\log \left\|D \phi_{t}(x) v\right\|}{t}=\Lambda_{k}
$$

for $1 \leq k \leq l, x \in B_{\text {reg }}$ and $v \in\left(\mathcal{C}_{k}\right)_{x}$. 
Lemma 3.1. For each $m \in \mathbf{M}_{\mathrm{erg}}(\Sigma)$ there is $I=I(m) \subsetneq\{1, \ldots, n\}$ such that the support supp $m$ of $m$ is contained in $A_{I}$.

Proof. By ergodicity of $m$ and invariance of all $\Sigma_{I}^{\circ}$, there is precisely one $I \subset$ $\{1, \ldots, n\}$ such that $m\left(\Sigma_{I}^{\circ}\right)=1$ and $m\left(\partial \Sigma_{I}\right)=0$. Further, as points from $\Sigma_{I}^{\circ} \backslash A_{I}$ are wandering (relative to $\Sigma$ ), one has $m\left(\Sigma_{I}^{\circ} \backslash A_{I}\right)=0$.

Fix $m \in \mathbf{M}_{\operatorname{erg}}\left(\Sigma_{I}\right)$ with $m\left(\Sigma_{I}^{\circ}\right)=1$, and put $\mathcal{B}:=\Sigma_{I} \times V_{I}, \mathcal{B}^{(i)}:=\Sigma_{I} \times V_{I \backslash i}, i \in I$. Evidently, $\mathcal{B}$ is a subbundle of $\mathcal{B}^{(i)}$ of codimension one. From the structure of system (E) it follows that the bundles $\mathcal{B}, \mathcal{B}^{(i)}$ are invariant. Denote by $\Lambda_{1}<\Lambda_{2} \cdots<\Lambda_{l}$ the Lyapunov exponents on $\mathcal{B}$ for the ergodic measure $m$ (we will call them the internal Lyapunov exponents for $m$ ). Among the Lyapunov exponents on $\mathcal{B}^{(i)}$ there is one (denoted by $\lambda^{(i)}(m)$ ) corresponding to the measurable linear set $\mathcal{C}_{k}^{(i)} \subset \mathcal{B}^{(i)}$ such that $\left(\mathcal{C}_{k}^{(i)}\right)_{x} \subsetneq V_{I}$ for $m$-a.e. $x \in \Sigma_{I}^{\circ}$. We will refer to $\lambda^{(i)}(m)$ as the $i$-th external Lyapunov exponent for $m$ (this terminology is modeled on Hofbauer's [6]).

The following result was essentially proved in the author's paper [10] (except for terminology).

Theorem 3.2. Assume that for each $m \in \mathbf{M}_{\text {erg }}(\partial \Sigma)$ all its external Lyapunov exponents are nonnegative. Then the following hold:

1. The carrying simplex $\Sigma$ is a $C^{1}$ submanifold-with-corners neatly embedded in C.

2. There are $\mu>0$ and an invariant one-dimensional subbundle $\mathcal{S}$ of $\Sigma \times V$ such that for each $m \in \mathbf{M}_{\mathrm{erg}}(\Sigma)$ one has

(a) $\Sigma \times V=T \Sigma \oplus \mathcal{S}$, where $T \Sigma$ denotes the tangent bundle of $\Sigma$, and $\left(\mathcal{C}_{1}\right)_{x} \subset$ $\mathcal{S}$ for $m$-a.e. $x \in \Sigma$.

(b) $\Lambda_{1}$ is internal.

(c) $\Lambda_{1} \leq-\mu$.

In the present section we make use of part 1 of Theorem 3.2 only.

In view of the above result, we need to prove only the following.

Proposition 3.3. Under the assumptions of Theorem A, for each $m \in \mathbf{M}_{\mathrm{erg}}(\partial \Sigma)$ all its external Lyapunov exponents are nonnegative.

Proof. Fix a measure $m \in \mathbf{M}_{\mathrm{erg}}\left(\Sigma_{I}\right)$ with $m\left(\Sigma_{I}^{\circ}\right)=1$, and an index $i \in I$. By Lemma 3.1, supp $m \subset A_{I}$. Take a regular point $x \in \operatorname{supp} m$ and a vector $v \in$ $\left(\mathcal{B}^{(i)}\right)_{x} \backslash V_{I}$ such that its $i$-th coordinate $v_{i}$ is positive. As $\left(\partial F_{i} / \partial x_{j}\right)\left(\phi_{t} x\right)=0$ for $j \neq i$, and $\left(\partial F_{i} / \partial x_{i}\right)\left(\phi_{t} x\right)=f_{i}\left(\phi_{t} x\right)$, it follows that the $i$-th coordinate $\left(D \phi_{t}(x) v\right)_{i}$ is the solution of the (nonautonomous) scalar linear ODE $\dot{\eta}=f_{i}\left(\phi_{t} x\right) \eta$ with initial condition $\eta(0)=v_{i}$. By strong competitiveness and Lemma 2.1, $f_{i}$ is positive on the compact invariant set $A_{I} \subset \Sigma_{I}$, hence there is $M>0$ such that $f_{i}\left(\phi_{t} x\right) \geq M$ for all $(t, x) \in \mathbb{R} \times A_{I}$. The standard theory of differential inequalities yields

$$
\liminf _{t \rightarrow \infty} \frac{\log \left(D \phi_{t}(x) v\right)_{i}}{t} \geq M
$$

$\|\cdot\|$ is the Euclidean norm on $\mathbb{R}^{n}$, therefore for all $t \in \mathbb{R}$ we have $\left\|D \phi_{t}(x) v\right\| \geq$ $\left(D \phi_{t}(x) v\right)_{i}$. By regularity of $x$ we derive

$$
\lim _{t \rightarrow \infty} \frac{\log \left\|D \phi_{t}(x) v\right\|}{t}=\lambda^{(i)}(m) \geq M>0 .
$$




\section{Proof of Theorem B}

We begin by stating a result which is an adaptation of a theorem of M. Benaïm.

Theorem 4.1. Assume that a $C^{k+1}, k=1, \ldots$, system (E) satisfies the following:

1. For each $m \in \mathbf{M}_{\mathrm{erg}}(\partial \Sigma)$ all external Lyapunov exponents are nonnegative.

2. There is $\eta>0$ such that for each $m \in \mathbf{M}_{\mathrm{erg}}(\Sigma)$ the inequality

$$
\Lambda_{1}(m)-(k+1) \Lambda_{2}(m)<-\eta
$$

holds, where $\Lambda_{1}(m)$ and $\Lambda_{2}(m)$ denote respectively the smallest and the second smallest Lyapunov exponents (on $\Sigma \times V$ ) for $m$.

Then the carrying simplex $\Sigma$ is a $C^{k+1}$ submanifold-with-corners.

Indication of proof. Theorem 3.2.2 asserts that the tangent bundle $T C$ restricted to $\Sigma$ invariantly decomposes as the Whitney sum $T \Sigma \oplus \mathcal{S}$, and for each $m \in \mathbf{M}_{\operatorname{erg}}(\Sigma)$ the smallest Lyapunov exponent $\Lambda_{1}(m) \leq-\mu<0$ is the exponential growth rate of a vector from $\mathcal{S}$, while any of the remaining Lyapunov exponents is the exponential growth rate of a vector tangent to $\Sigma$. This, together with (4.1), gives, with the help of Prop. 3.3 in [1] (based on a result of S. Schreiber [11]), that there are $c \geq 1$, $\alpha>0$ and $\beta>0$ such that

$$
\left\|D \phi_{t}(x) v\right\| \leq c e^{-\alpha t}\|v\| \quad \text { for } t \geq 0,(x, v) \in \mathcal{S},
$$

and

$$
\frac{\left\|D \phi_{t}(x) v\right\|}{\left\|D \phi_{t}(x) w\right\|^{k+1}} \leq c e^{-\beta t} \quad \text { for } t \geq 0, x \in \Sigma, v \in(\mathcal{S})_{x}, w \in T_{x} \Sigma \backslash\{0\}
$$

The rest of the proof consists in applying the $C^{k+1}$ section theorem of Hirsch, Pugh and Shub [5], as in the proof of Thm. 3.4 in [1].

As a consequence of the above theorem and Proposition 3.3, we will have Theorem $\mathrm{B}$ once we prove the following.

Proposition 4.2. Assume that a $C^{k+1}$ system (E) satisfies (A) and (C). Then there exists $\eta>0$ such that for each $m \in \mathbf{M}_{\mathrm{erg}}(\Sigma)$ the inequality (4.1) holds.

Proof. Take $m \in \mathbf{M}_{\text {erg }}(\Sigma)$, and let $I \subset\{1, \ldots, n\}$ be such that $m\left(\Sigma_{I}^{\circ}\right)=1$. From Lemma 3.1 we have supp $m \subset A_{I}$. By results contained in Sections 3 and 4 of [1], it follows that under assumption $(\mathrm{C})$ there is $\eta_{I}>0$ such that

$$
\Lambda_{1}^{*}(m)-(k+1) \Lambda_{2}^{*}(m)<-\eta_{I}
$$

for all $m$ supported on $A_{I}$, where $\Lambda_{1}^{*}(m)$ [resp. $\left.\Lambda_{2}^{*}(m)\right]$ stands for the smallest [resp. second smallest] internal Lyapunov exponent for $m$. Theorem 3.2.2 gives $\Lambda_{1}^{*}(m)=\Lambda_{1}(m)$. Denote by $\lambda_{\text {min }}$ the smallest external Lyapunov exponent for $m$. If $\lambda_{\min } \geq \Lambda_{2}^{*}(m)$, then $\Lambda_{2}^{*}(m)=\Lambda_{2}(m)$ and the inequality (4.1) is satisfied with $\eta_{I}$. Assume that $\lambda_{\min }<\Lambda_{2}^{*}(m)$. Applying Theorem 3.2.2 and Proposition 3.3, we obtain $\Lambda_{1}(m) \leq-\mu<0 \leq \lambda_{\min }=\Lambda_{2}(m)$. Consequently, $\Lambda_{1}(m) \leq-\mu<0 \leq$ $(k+1) \Lambda_{2}(m)$. It suffices to put

$$
\eta:=\min \left\{\mu, \eta_{I}: I \subset\{1, \ldots, n\}, \operatorname{card} I \leq n-1\right\} .
$$




\section{Discussion}

Remark 5.1. In formulating our results, we preferred that the assumptions be easily tractable rather than the weakest possible or that they cover a wide range of applications. In fact, they can be substantially weakened, as the following example shows.

A celebrated Lotka-Volterra system due to May and Leonard [9] has the form

$$
\begin{aligned}
& \dot{x}_{1}=x_{1}\left(1-x_{1}-\alpha x_{2}-\beta x_{3}\right), \\
& \dot{x}_{2}=x_{2}\left(1-\beta x_{1}-x_{2}-\alpha x_{3}\right), \\
& \dot{x}_{3}=x_{3}\left(1-\alpha x_{1}-\beta x_{2}-x_{3}\right),
\end{aligned}
$$

with $0<\beta<1<\alpha$ and $\alpha+\beta>2$. It is easily verified that (5.1) is dissipative, totally competitive and has five rest points on $C$ : 0 (repelling), $y$ with $y_{i}=1 /(1+$ $\alpha+\beta)$ and three axial ones $x^{(i)}$ with $x_{i}^{(i)}=1$. Furthermore, $\partial \Sigma$ is an attractor relative to $\Sigma$ with $\{y\}$ as its complementary repeller (see pp. 67-68 in the book [7] by Hofbauer and Sigmund). As a consequence, $\mathbf{M}_{\operatorname{erg}}(\Sigma)=\left\{\delta_{y}, \delta_{x^{(1)}}, \delta_{x^{(2)}}, \delta_{x^{(3)}}\right\}$. The Lyapunov exponents for the Dirac delta on a rest point $x$ are simply the real parts of the eigenvalues of $D F(x)$. At $y$ the smallest Lyapunov exponent is negative and the remaining ones are positive (see [7]). A simple calculation shows that at an axial rest point the unique internal Lyapunov exponent equals -1 and the external exponents are $1-\beta>0$ and $1-\alpha<0$.

Assume now that $\alpha<2$. Then for each ergodic measure on $\Sigma$ the smallest Lyapunov exponent -1 is internal. Applying ideas of the author's earlier paper [10] we prove that the carrying simplex $\Sigma$ for (5.1) is a $C^{1}$ submanifold-with-corners. Observe that if $1<\alpha<1+1 / l$ for some $l=2, \ldots$, then $-1-l(1-\alpha)$ is negative and one can deduce along the lines of the proof of Theorem 4.1 to conclude that $\Sigma$ is of class $C^{l}$.

Remark 5.2. The systems (E) satisfying (A) [resp. (A) and (C)] are robust in the sense that if we perturb $f$ in a neighborhood of $\Sigma$ in the $C^{1}$ [resp. in the $C^{k+1}$ ] topology, then the perturbed system possesses a carrying simplex of class $C^{1}$ [resp. $C^{k+1}$ ] (and each of its subsystems $(\mathrm{E})_{I}$ is permanent). This can be proved by reasoning similar to that in the proof of Cor. 4.3 in [1].

Remark 5.3. In principle, results contained in Section 3 should carry over to the case where we allow $f$ to depend periodically on $t$, although finding an analog of (A) might be tricky (for time-periodic Lotka-Volterra strongly competitive systems, compare e.g. [14]).

\section{REFERENCES}

[1] M. Benaïm, On invariant hypersurfaces of strongly monotone maps, J. Differential Equations 137 (1997), 302-319. MR 98d:58114

[2] M. Gobbino and M. Sardella, On the connectedness of attractors for dynamical systems, J. Differential Equations 133 (1997), 1-14. CMP 97:06

[3] J. K. Hale, Asymptotic behavior of dissipative systems, Math. Surveys Monogr. 25, American Mathematical Society, Providence, R.I., 1988. MR 89g:58059

[4] M. W. Hirsch, Systems of differential equations which are competitive or cooperative. III. Competing species, Nonlinearity 1 (1988), 51-71. MR 90d:58070

[5] M. W. Hirsch, C. C. Pugh and M. Shub Invariant manifolds, Lecture Notes in Math. 583, Springer, Berlin-New York, 1977. MR 58:18595

[6] J. Hofbauer, An index theorem for dissipative semiflows, Rocky Mountain J. Math. 20 (1990), 1017-1031. MR 92b:58203 
[7] J. Hofbauer and K. Sigmund, The theory of evolution and dynamical systems, London Mathematical Society Student Texts 7, Cambridge University Press, Cambridge, 1988. MR 91h:92019

[8] R. Mañé, Ergodic theory and differentiable dynamics (translated from the Portuguese by S. Levy), Ergeb. Math. Grenzgeb. (3) 8, Springer, Berlin-New York, 1987. MR 88c:58040

[9] R. M. May and W. J. Leonard, Nonlinear aspects of competition between three species, SIAM J. Appl. Math. 29 (1975), 243-253. MR 52:12853

[10] J. Mierczyński, The $C^{1}$ property of carrying simplices for a class of competitive systems of ODEs, J. Differential Equations 111 (1994), 385-409. MR 95g:34066

[11] S. J. Schreiber, Expansion rates and Lyapunov exponents, Discrete Contin. Dynam. Systems 3 (1997), 433-438. MR 98c:58096

[12] H. L. Smith, Monotone dynamical systems. An introduction to the theory of competitive and cooperative systems, Math. Surveys Monogr. 41, Amer. Math. Soc., Providence, R.I., 1995. MR 96c:34002

[13] I. Terešćák, Dynamics of $C^{1}$ smooth strongly monotone discrete-time dynamical systems, preprint.

[14] A. Tineo, An iterative scheme for the $N$-competing species problem, J. Differential Equations 116 (1995), 1-15. MR 95m:92023

[15] M. L. Zeeman, Hopf bifurcations in competitive three-dimensional Lotka-Volterra systems, Dynam. Stability Systems 8 (1993), 189-217. MR 94j:34044

Institute of Mathematics, WrocŁaw University of Technology, Wybrzeże WyspiańSKiego 27, PL-50-370 WrocŁaW, Poland

E-mail address: mierczyn@banach.im.pwr.wroc.pl 